Proyecciones Journal of Mathematics

Vol. 36, No 2, pp. 299-306, June 2017.

Universidad Católica del Norte

Antofagasta - Chile

\title{
On the toral rank conjecture and some consequences
}

\author{
Hassan Aaya \\ Mohamed Anas Hilali \\ Mohamed Rachid Hilali \\ and \\ Tarik Jawad \\ University Hassan II, Morocco \\ Received: May 2016. Accepted: March 2017
}

\begin{abstract}
The aim of this work is to improve the lower bound of the Puppe inequality. His theorem [15, Theorem 1.1] states that the sum of all Betti numbers of a well-behaved space $X$ is at least equal to $2 n$, where $n$ is rank of an n-torus $T^{n}$ acting almost freely on $X$.
\end{abstract}




\section{Introduction}

The well-known Halperin conjecture [8, p. 271] about torus actions on topological spaces is behind many works in mathematics like the Hilali conjecture [12] and the inequality of Puppe [15 Theorem 1.1]: If $\mathrm{X}$ is a space on which an n-torus acts, we say the action is almost-free if each isotropy subgroup is finite. The largest integer $\mathrm{n} \geq 1$ for which $\mathrm{X}$ admits an almost free $\mathrm{n}$-torus is called the toral rank of $\mathrm{X}$ and denoted $r k(X)$. If $\mathrm{X}$ does not admit any almost free torus action, then $r k(X)=0$. Unfortunately $r k(X)$ is not a homotopy invariant and is quite difficult to compute. To obtain a homotopy invariant, we introduce the rational toral rank, $r k_{0}(X)$ that is, the maximum of $r k(Y)$ among all finite $\mathrm{CW}$ complexes $\mathrm{Y}$ in the same rational homotopy type as $\mathrm{X}$.

Conjecture (The Toral rank conjecture).

If $X$ is simply connected, then $\operatorname{dim} H^{*}(X ; \mathbf{Q}) \geq 2^{r k_{0}(X)}$.

Conjecture (The Hilali conjecture).

If $X$ is elliptic and simply connected, then $\operatorname{dim}\left(\pi_{*}(X) \otimes \mathbf{Q}\right) \leq \operatorname{dim}\left(H^{*}(X ; \mathbf{Q})\right)$.

Theorem 1.1 (Puppe Inequality).

If $X$ is simply connected, then $\operatorname{dim} H^{*}(X ; \mathbf{Q}) \geq 2 r k_{0}(X)$.

In the present paper we give in section 2 an optimised proof of the theorem 1.1, and in section 3 we establish a new theorem with an improvement of the lower bound of the Puppe inequality.

\section{Acknowledgments}

The authors are grateful to Aziz El Kacimi for pointing out a mistake in the original version of the paper and for suggesting examples.

\section{New proof of the theorem 1.1}

Let $\mathrm{X}$ be a simply connected topological space with an almost free $T^{n}$ action. We denote $r k_{0}(X)=n$ and we suppose that $n \neq 0$.

The Sullivan minimal model of the classifying space $B_{T^{n}}$ of the Lie group $T^{n}$ is a polynomial ring denoted here $R$, with the following form:

$R=\left(\Lambda\left(t_{1}, \cdots, t_{n}\right), 0\right)$ with $\operatorname{deg}\left(t_{i}\right)=2$. 
The associated Borel fibration [5, p. 53] of this action is:

$$
X \longrightarrow X_{T^{n}} \longrightarrow B_{T^{n}}
$$

According to Brown [6], there exists a complex of differential $R$-modules $\left(R \otimes H^{*}(X ; \mathbf{Q}), \Delta\right)$ with a quasi-isomorphism of R-modules :

$$
\varrho:\left(R \otimes\left(H^{*}(X ; \mathbf{Q}), \Delta\right) \rightarrow A_{P L}\left(X_{T^{n}}\right)\right.
$$

Let $\beta=\left\{\alpha_{1}, \cdots, \alpha_{p}, \alpha_{p+1}, \cdots, \alpha_{2 p}\right\}$ be a basis of $H^{*}(X, \mathbf{Q})$ such that:

$$
\left|\alpha_{i}\right| \text { is odd for : } \quad 1 \leq i \leq p
$$$$
\left|\alpha_{i}\right| \text { is even for : } p+1 \leq i \leq 2 p
$$

The differential $\Delta$ can be written for $i, 1 \leq i \leq p$

$$
\Delta\left(1 \otimes \alpha_{i}\right)=P_{i} \otimes 1+\sum_{j=1}^{p} t_{i j} \alpha_{j+p}
$$

where $P_{i}$ is a homogeneous polynomial in $\left(t_{1}, \cdots, t_{n}\right)$.

Lets consider the $p \times p$ matrix over $R, M=\left(t_{i j}\right)_{1 \leq i, j \leq p}$, and lets denote

$$
I=\left\{\sum_{j=1}^{p} a_{i} P_{i} / M\left[\begin{array}{c}
a_{1} \\
\vdots \\
a_{p}
\end{array}\right]=0\right\}
$$

I is an ideal of $\mathrm{R}$, and we have the following diagram:

$$
\begin{array}{ccc}
(R, 0) & \stackrel{i}{\rightarrow} & \left(R \otimes H^{*}(X, \mathbf{Q}), \Delta\right) \\
\downarrow p & & \bar{i} \nearrow \\
(\bar{R}, 0) & &
\end{array}
$$

where $i$ is the canonical injection and $p$ is the canonical surjection, $\bar{i}$ is induced by passing $i$ to the quotient.

By passing to cohomology the diagram hereinabove induces the following diagram:

$$
\begin{array}{ccc}
(R, 0) & \stackrel{i^{*}}{\rightarrow} & H\left(R \otimes H^{*}(X, \mathbf{Q}), \Delta\right) \\
\downarrow & & i^{*} \nearrow \\
(\bar{R}, 0) &
\end{array}
$$


$\overline{i^{*}}$ is injective because if $\bar{a} \in \bar{R}$ such that $\overline{i^{*}}(\bar{a})=0=i^{*}(a)$ then

$$
\begin{aligned}
& a \otimes 1=\Delta\left(\sum_{i=1}^{p} a_{i} \alpha_{i}\right) \\
& =\sum_{i=1}^{p} a_{i} P_{i} \otimes 1+\sum_{i=1}^{p} a_{i}\left(\sum_{i=1}^{p} t_{i j} \alpha_{j+p}\right) \\
& =\sum_{i=1}^{p} a_{i} P_{i} \otimes 1+\sum_{i=1}^{p} a_{i}\left(\left(\sum_{i=1}^{p} a_{i} t_{i j}\right) \alpha_{j+p}\right)
\end{aligned}
$$

Then $a=\sum_{i=1}^{p} a_{i} t_{i j}$ and $\sum_{i=1}^{p} a t_{i j}=0 \forall i, 1 \leq i \leq p$.

Hence $a \in I$ so $\bar{a}=0$.

Therefore $\operatorname{dim} \bar{R} \leq \operatorname{dim} H^{*}\left(X_{T^{n}} ; \mathbf{Q}\right)<\infty$.

And $\operatorname{dim} \bar{R} \geq n$ since $\left\{\overline{t_{1}}, \cdots, \overline{t_{n}}\right\}$ is a free family in $\bar{R}$.

In another hand since $M$ is a square matrix of order $p$ over the ring $R$ then $I$ is a free $R$-module of dimension $N \leq p$.

We have $\operatorname{dim} R / I<\infty$ so $\operatorname{dim} I=N \geq n$ witch implies that $\operatorname{dim} H^{*}(X, \mathbf{Q}) \geq 2 n$.

\section{An improvement of the lower bound of Puppe inequality}

\subsection{The main theorem}

Let $\mathrm{X}$ be a simply connected topological space with an almost free $T^{n}$ action, such that $\operatorname{dim} H^{*}(X ; \mathbf{C})<\infty$.

The associated Borel fibration [5, p. 53] of this action is:

$$
X \longrightarrow X_{T^{n}} \longrightarrow B_{T^{n}}
$$

$X_{T^{n}}$ has the Hirsch-Brown minimal model

$D_{T^{n}}=\left(H^{*}\left(B_{T^{n}} ; \mathbf{C}\right) \otimes H^{*}(X ; \mathbf{C}) ; \tilde{d}\right)$ as a $H^{*}\left(B_{T^{n}} ; \mathbf{C}\right)$-Module $[2$, Section $1.3]$.

We define an increasing filtration $F_{q}$ on $H^{*}(X ; \mathbf{C})$ by:

$F_{-1}=0$

$F_{q}=\left(\left.\tilde{d}(x)\right|_{H^{*}(X ; \mathbf{C})}\right)^{-1}\left(H^{*}\left(B_{T^{n}} ; \mathbf{C}\right) \otimes F_{q-1}\right)$

The length $l(X)$ of $H^{*}(X, \mathbf{C})$ is defined by :

$$
l=\inf \left\{q \in \mathbf{N} / F_{q}=H^{*}(X, \mathbf{Q})\right\} .
$$


We use the evaluation at $\alpha=\left(\alpha_{1}, \cdots, \alpha_{n}\right)$ to define the new space:

$$
D_{T^{n}}(X)^{\alpha}=\mathbf{C} \otimes_{H^{*}\left(B_{T^{n}} ; \mathbf{C}\right)} D_{T^{n}}(X)
$$

where the structure of this $H^{*}(X ; \mathbf{C})$-module is defined by the map:

$$
\begin{aligned}
& H^{*}\left(B_{T^{n}} ; \mathbf{C}\right) \rightarrow \mathbf{C} \\
& t_{i} \quad \mapsto \alpha_{i}
\end{aligned}
$$

we know by $[3$, theorem $4-1]$, that for $\alpha \neq(0, \cdots, 0)$ we have:

$H^{*}\left(D_{T^{n}}(X)^{\alpha}, \tilde{d}_{\alpha}\right)=0$.

The coboundary $\tilde{d}_{\alpha}$ is given by $\tilde{d}$ evaluated at $\alpha \in \mathbf{C}([2, \mathrm{p} .26])$,

Now for every $q, 0 \leq q \leq l$, we define $A_{q}$ to be a complement of $F_{q-1}$ in $F_{q}:$

$$
F_{q}=A_{q} \oplus F_{q-1}
$$

The main result of this article is an improvement of the lower bound of Puppe inequality expressed in the following theorem:

Theorem 3.1. Let $X$ be a simply connected topological space with an almost free $T^{n}$-action, we denote $n=r k_{0}(X)$ for $n \geq 4$ we always have $\operatorname{dim} H^{*}(X ; \mathbf{Q}) \geq 3 n-2$

The proof of this theorem is based on the following lemma:

Lemma 3.2. Under the same conditions as the theorem above one has: $\operatorname{dim} A_{1} \geq n$.

Definition 3.3.[17, vol 1, p. 90] By $\mathbf{P}^{n}$ we denote $n$-dimensional projective space over $\mathbf{C}$. A projective algebraic variety $V$ is an algebraic subset of $\mathbf{P}^{n}$, that is, the zero-set of some homogeneous polynomials $f_{i}, i \in I$, in the homogeneous coordinates $\left(x_{0}, \cdots, x_{n}\right)$ of $\mathbf{P}^{n}: V=\left\{\left(x_{0}, \cdots, x_{n}\right) \mid f_{i}\left(x_{0}, \cdots, x_{n}\right)=\right.$ $0, i \in I\}$.

Proof of the lemma 3.2 According to Puppe [16, p. 7], we know that $l \geq n$, and $\operatorname{dim} A_{q} \geq 2$, for every $q, 1 \leq q \leq l-1$.

Let $\left\{a_{1}, \cdots, a_{r}\right\}$ and $\left\{b_{1}, \cdots, b_{s}\right\}$ be two bases of $A_{1}$ and $A_{0}$. For each $i, \quad 1 \leq i \leq r$ we can write:

$$
\tilde{d}\left(a_{i}\right)=p_{i} b_{1}+\omega_{i}
$$

where $p_{i}$ are homogeneous polynomials on $t_{1}, \cdots, t_{n}$ and $\omega_{i}$ is a linear composition of $b_{2}, \cdots, b_{s}$ over $\mathbf{Q}\left[t_{1}, \cdots, t_{n}\right]$. If we suppose that $r<n$, then the 
algebraic variety $V\left(p_{1}, \cdots, p_{r}\right)$ is different from $\{(0, \cdots, 0)\}$. Hence we can take $\alpha \in V\left(p_{1}, \cdots, p_{r}\right) \backslash\{(0, \cdots, 0)\}$ such that:

$\tilde{d}_{\alpha} b_{1}=0$ (because $\left.F_{0}=A_{0}=\operatorname{ker}(\tilde{d})\right)$.

$\tilde{d}_{\alpha} a_{i}=\omega_{i}$ for $1 \leq i \leq r$.

This shows us that the $\tilde{d}_{\alpha^{-}}$cocyle $b_{1}$ is not a zero in $H^{*}\left(D_{T^{n}}(X)^{\alpha}, \tilde{d}_{\alpha}\right)$ which is absurd.

Proof of the Theorem 3.1 Let's denote $m_{q}=\operatorname{dim} A_{q}, 0 \leq q \leq l$. Then we have $\operatorname{dim} H^{*}(X ; \mathbf{Q})=\sum_{q=0}^{l} m_{q}$.

One has:

$\operatorname{dim} H^{*}(X ; \mathbf{Q}) \geq m_{0}+m_{1}+m_{l}+\sum_{q=2}^{l-1} m_{q}$

$\geq 2+n+2(n-1)$.

$\geq 3 n-2$.

\subsection{Examples}

Remark 3.4. The theorem 3.1 gives a measurement of the obstruction of a manifold to have an almost free $T^{n}$-action, for example a compact simply connected manifold $M$ with the sum of it's Betti numbers $<3 n-2$ can't have an almost free $T^{n}$-action.

Example 3.5. The toral rank of the manifold $M=\left(\mathbf{S}^{2 n+1}\right)^{r}$ is equal to $r$ and the sum of it's Betti numbers is equal to $2^{r}$ [8, p. 284], We have $\operatorname{dim} H^{*}\left(\left(\mathbf{S}^{2 n+1}\right)^{4} ; \mathbf{Q}\right)=16<3 \times 7-2=19$ so $\left(\mathbf{S}^{2 n+1}\right)^{4}$ can't have an almost free $T^{7}$-action.

Remark 3.6. In 2012 M. Amann [4, Theorem A] established the following result:

Theorem A. If an $n$-torus $T$ acts almost freely on a finite-dimensional paracompact Hausdorf space $X$, then $\operatorname{dim} H^{*}(X ; \mathbf{Q}) \geq 2(n+[n / 3])$

$X$ may be taken to be a finite $\mathrm{CW}$-complex or a compact manifold.

It's clear that starting from $n=7$ the theorem 3.1 gives a greater lower bound than theorem A . 


\section{References}

[1] C. Allday and S. Halperin, Lie group actions on spaces of finite rank, Quart. J. Math. Oxford (2) 29, pp. 63-76, (1978).

[2] C. Allday and V. Puppe, Cohomological methods in transformation groups, volume 32 of Cambridge Studies in Advanced Mathematics. Cambridge University Press, Cambridge, (1993).

[3] C. Allday and V. Puppe, On the localization theorem at the cochain level and free torus actions, Algebraic topology Göttingen 84, Procedings, Springer lect. Notes in Math 1172, pp. 1-16, (1985).

[4] M. Amann, Cohomological consequences of almost free torus actions arXiv:1204.6276, Vol. 1 27, April (2012).

[5] A. Borel, Seminar on transformation groups Ann. of math Studies $n^{\circ}$ 46. Princeton New Jersey.

[6] E. H. Brown, Twisted tensor product I, Ann. of Math vol. 69, pp. 223246, (1959).

[7] Y. Félix, S. Halperin, and J.-C. Thomas, Rational homotopy theory, volume 205 of Graduate Texts in Mathematics. Springer-Verlag, New Yo, (2001).

[8] Y. Félix, J. Oprea, and D. Tanré, Algebraic models in geometry, volume 17 of Oxford Graduate Texts in Mathematics. Oxford University Press, Oxford, (2008).

[9] S. Halperin, Finiteness in the minimal models of Sullivan, Trans. A. M. S. 230, pp. 173-199, (1977).

[10] S. Halperin, Rational homotopy and torus actions, London Math. Soc. Lecture Note Series 93, Cambridge Univ. Press, pp. 293-306, (1985).

[11] M. R. Hilali, Sur la conjecture de Halperin relative au rang torique. Bull. Belg. Math. Soc. Simon Stevin 7, No. 2, pp. 221-227, (2000).

[12] M. R. Hilali, Actions du tore $T^{n}$ sur les espaces simplement connexes. Thèse à l'Université catholique de Louvain, (1990). 
[13] W. Y. Hsiang, Cohomology theory of topological transformation groups, Berlin-Heidelberg-New York, Springer, (1975).

[14] I. M. James, reduced product spaces, Ann. of math 82, pp. 170-197, (1995).

[15] V. Puppe, Multiplicative aspects of the Halperin-Carlsson conjecture, Georgian Mathematical Journal, (2009), 16:2, pp. 369379, arXiv 0811.3517.

[16] V. Puppe, On the torus rank of topological spaces, Proceding Baker, (1987).

[17] I. R. Shafarevich, Basic Algebraic Geometry, 2 Vols., Springer, (1994).

[18] Yu. Ustinovskii, On almost free torus actions and Horroks conjecture, (2012), arXiv 1203.3685v2.

\section{Hassan Aaya}

University Hassan II, Casablanca

Morocco

e-mail : aaya.hassan@gmail.com

\section{Mohamed Anas Hilali}

University Hassan II, Casablanca

Morocco

e-mail : moahilali@gmail.com

\section{Mohamed Rachid Hilali}

University Hassan II,

Casablanca

Morocco

e-mail : rhilali@hotmail.fr

and

\section{Tarik Jawad}

University Hassan II,

Casablanca

Morocco

e-mail : j_tar@hotmail.fr 